\title{
Quake Delirium EEG
}

\section{A Pilot Study Regarding Biofeedback-Driven Visual Effects in a Computer Game}

\author{
Jonathan Weinel, Stuart Cunningham, Nathan Roberts, Darryl Griffiths \& Shaun Roberts \\ Creative and Applied Research for the Digital Society (CARDS) \\ Glyndŵr University, UK \\ \{j.weinel | s.cunningham | n.roberts | griffiths.d | roberts.s\}@glyndwr.ac.uk
}

\begin{abstract}
Altered states of consciousness (ASC) can be represented in video games through appropriate use of sound and computer graphics. Our research seeks to establish systematic methods for simulating ASC using computer sound and graphics, to improve the realism of ASC representations in video game engines. Quake Delirium is a prototype 'ASC Simulation' that we have created by modifying the video game Quake. Through automation of various graphical parameters that represent the conscious state of the game character, hallucinatory ASC are represented. While the initial version of Quake Delirium utilised a pre-determined automation path to produce these changes, we propose that immersion may be improved by providing the user with a 'passive' method of control, using a brain-computer interface (BCI). In this initial trial, we explore the use of a consumer-grade electroencephalograph (EEG) headset for this purpose.
\end{abstract}

Keywords-Altered States of Consciousness; Video Games; Brain-Computer Interface (BCI)

\section{INTRODUCTION}

The principal aim of this research is to advance methods through which hallucinatory experiences can be represented in computer games. In recent years video game developers have sought to provide video gaming experiences that are more cinematic and realistic than before, particularly within certain genres such as 'firstperson shooter' (FPS) action games. Along with this drive for realism we have begun to see increased levels of sophistication regarding the way in which subjective perceptual states are represented within these games. In some cases, the subjective experience of hallucination has been represented using a combination of visual effects, audio and music; this is seen in Dead Space 2 (2011), Far Cry 3 (2012) and Grand Theft Auto V (2013) for example. The games that exhibit these hallucinatory sequences have been discussed by Weinel [1], Demarque and Lima [2] and on various internet blogs and news sites such as Blake [3].

In order to consider how first-person perspective representations of conscious experience typically operate within a video game context, we may consider the conceptual diagram presented in Fig. 1. Computer graphics are used to represent the 3D geometry of the gaming environment, from the first-person perspective of the game character, in a way that approximates how we see the world around us. This usually achieved with a game camera that is mounted above the body of a player (which in practice is often a simple capsule primitive in first-person shooters, where it does not need to be displayed). Computer audio represents the game character's first-person aural experience of the virtual environment. This is typically achieved using 3D sound, which presents sound in stereo according to the game character's relative location to sound sources within the virtual environment. Typically the amplitudes of sound sources are attenuated based on the distance of the player from them, and Doppler effects may also be provided for moving sound sources. The realism of $3 \mathrm{D}$ sound currently lags somewhat behind other aspects of game realism such as graphics, but in the future we can expect to see 3D sound which also simulates other aspects of our auditory perception such as inter-aural time differences between sources, and the filtering of sounds due to head masking of the game character. Lastly, Fig. 1 also indicates the property of time, which in the virtual environment usually passes in a roughly equivalent manner to that which we experience in real life (the exceptions to this are games that accelerate the passing of day and night or allow the character to walk unrealistically quickly in order to prevent player boredom). The combination of features such as these facilitate a representation of the subjective, first-person perspective experience of consciousness, of the virtual game character, which is relayed to the human player via a visual display (e.g. computer monitor) and loudspeakers. 


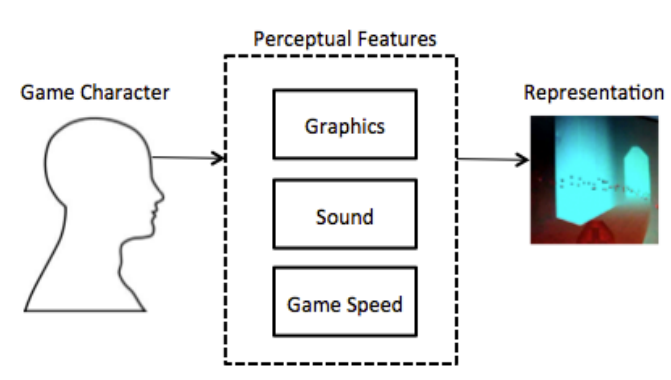

Fig. 1. Indicating the main game engine properties that mimetically represent phenomenological properties of consciousness, with regards to a video game character.

If we accept that the representation of a virtual, subjective experience of consciousness is achieved using the properties of sound, graphics and time, we may consider how altering aspects of the way in which these properties present that conscious experience could allow for variation in the representation of subjective sensory experience. For example, modifying the graphical properties that represent vision could enable us to simulate visual impairment (e.g. short sightedness); filtering of modification to the audio could reflect auditory impairment (e.g. tinnitus); and manipulation of time properties could reflect situations where time subjectively seems to pass more quickly or slowly than usual.

By modifying these properties we can simulate different ways of perceiving in the virtual game environment, such as may be typical in hallucinatory altered state of consciousness. The game engine may then allow us to represent the subjective experience of consciousness, for various states of consciousness (such as ASCs). In this way, we can provide an 'ASC Simulation'.

\section{ASC SIMULATION}

Quake Delirium is an early prototype 'ASC Simulation' which was developed previously [1, 4] (Fig. 2). Quake Delirium functions by automating various game parameters that are usually static, such as those related to graphical features and game speed. Automating these parameters in time enables a basic representation of hallucinatory ASC. These parameters are placed in a constant state of flux, as this is considered appropriate to imitate the constant change that characterise hallucinatory ASC. Quake Delirium also includes a corresponding electroacoustic soundtrack (discussed in [1]).

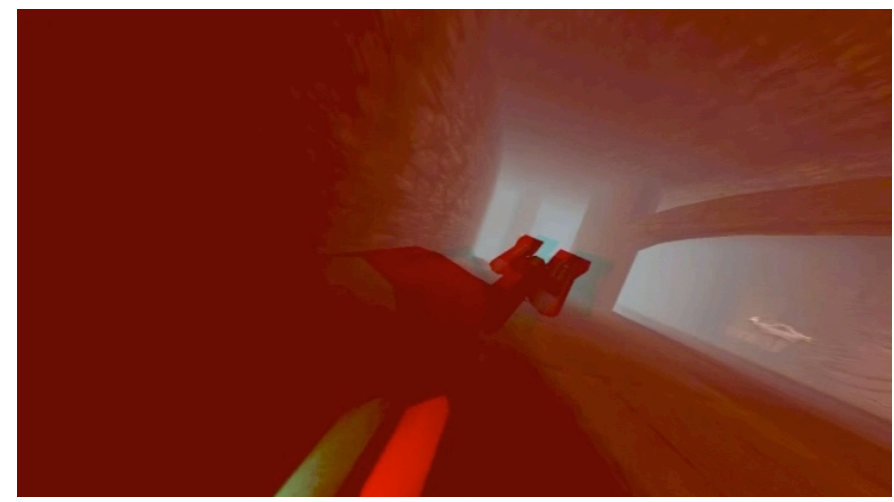

Fig. 2. Screen capture from Quake Delirium, showing an altered representation of the game environment, as produced by the modification.

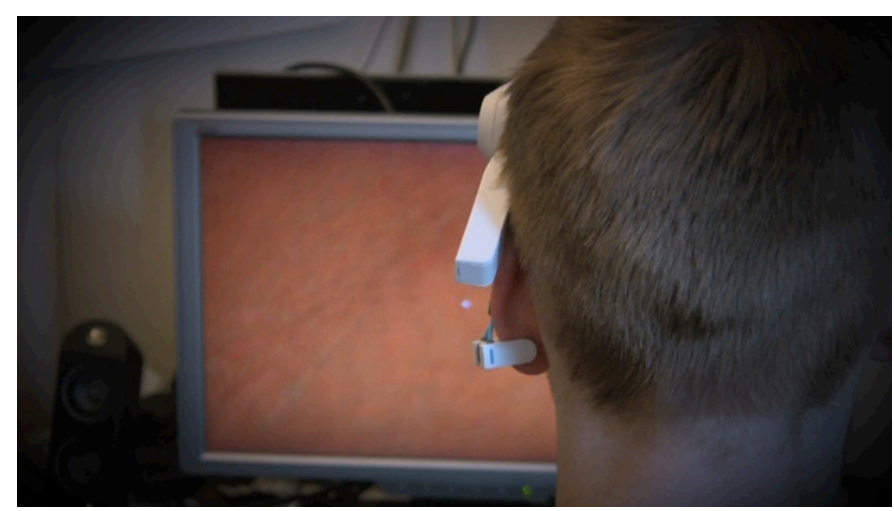

Fig. 3. Quake Delirium, with a NeuroSky MindWave EEG headset linked as a passive controller.

The original version of Quake Delirium created these automated changes using pre-determined automation envelopes. However, it was proposed that a more immersive and embodied experience may be provided by linking these to the person playing the game through the use of biofeedback. The type of interaction sought can be described as either 'passive' or 'reactive' [5], since the player does not need to willfully control these effects ('active' control); they should simply experience being connected to them in some meaningful way.

In this initial trial, we explore the possibility of using a consumer-grade electroencephalograph (EEG) headset as a controller for this purpose. Through the headset, the player's brain activity provides a control signal that is mapped to affect various psychedelic visual effects (Fig. 3). In our pilot study we compare ASC systems with and without the use of an EEG headset as a control device, in order to explore the extent to which the headset enables improved levels of immersion and realism in the representation of ASC.

\section{Methodology}

The experiment was conducted in our usability lab under controlled conditions. We utilised ten 
participants, recruited from the student population, who had some basic experience of video games and audio technology. A training phase was provided in which the participants were familiarised with the Quake Delirium system under normal conditions without the modification. Following this we compared two variations of the ASC system for 4 minutes each.

In Part A (Fig. 4, a), the basic Quake Delirium system was used, where the changes to graphical and game parameters follow a pre-determined automation sequence.

In Part B (Fig. 4, b), the Quake Delirium system was used with the EEG headset as a control device for certain parameters. The headset used is a NeuroSky Mindwave, an inexpensive consumer-grade EEG headset that uses dry sensors. This headset was chosen as it provides a convenient EEG solution that could easily be adapted for use in computer games. In Part B, the real-time signals from the EEG headset provide control signals that change various visual effects parameters in time, thus linking brain activity to psychedelic visual effects. Other parameters remain automated according to a predetermined sequence.

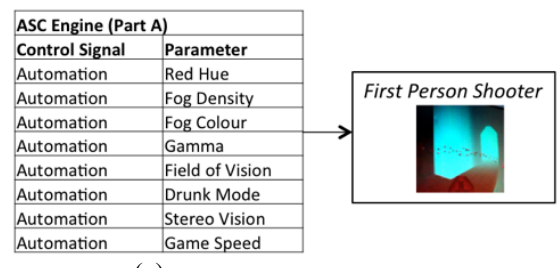

(a)

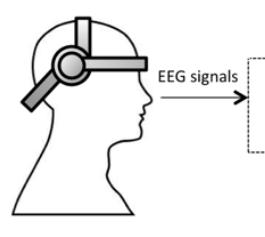

ASC Engine (Part B) Control Signal Parameter EEG: Attention Red Hue

EEG: Meditation Fog Density

EEG: Delta Fog Colour

\begin{tabular}{ll} 
EEG: Theta & Gamma \\
\hline Algar
\end{tabular}

\begin{tabular}{l|l|}
\hline EEG: Theta & Gamma \\
\hline Automation & Field of Vision \\
\hline
\end{tabular} \begin{tabular}{|l|l|}
\hline Automation & Field of Vision \\
\hline Automation & Drunk Mode \\
\hline Auton & Steo Vision \\
\hline
\end{tabular} Automation Stereo Vision Automation Game Speed

(b)

Fig. 4. Diagrams showing control mapping for game parameters in the Part A (a) and Part B (b) systems. Both systems enable perceptual features to change over time in order to simulate ASC effects. The Part B system introduces an EEG headset to provide a control signal for several parameters, linking brain activity to the psychedelic simulation.

Participants were asked to complete a short questionnaire regarding their experience of the systems, consisting of statements about their perceptions of Quake Delirium scored on a semantic differential scale of 1 to 5. We therefore hoped to show if the use of the EEG headset as a control device was perceived to improve the quality and immersion of the ASC simulation system; in effect, whether it provided a useful, tangible passive (or possibly reactive) connection for the user.

\section{RESULTS}

The initial trial indicated that both systems were perceived as 'psychedelic' by our participants. However, the use of the EEG headset showed no clear benefit between Part A and Part B in terms of immersion, $t(9)=-1.077, p>.05$, or how psychedelic, $t(9)=0.612, p>.05$, the system was perceived as being. From a previous study carried out with the same EEG headset, we did not expect our participants would be able to wilfully control the signals produced by the headset (active control) [6]. Feedback from our participants suggests that some sense of a connection between the headset and the graphical changes is perceptible, however the nature of this relationship appears to be ambiguous for the user at present. The following statements from the participants, are illustrative of the impression:

"I felt like some of the actions such as fog may have been created by the brainwave detection."

"The immersion was better with the EEG but I didn't feel that effects changed in relation with my brain activity."

TABLE I. THE RESUlTS OF USER TESTING FOR QUAKE DELIRIUM

\begin{tabular}{|l|r|r|}
\hline Question & Mean Response & \multicolumn{2}{|c|}{ St. Dev } \\
\hline Part A & 3.9 & 0.99 \\
\hline $\begin{array}{l}\text { To what extent did you find the visual effects in } \\
\text { the game psychedelic? }\end{array}$ & 3.3 & 1.16 \\
\hline How immersive did you find the video game? & 3.7 & 0.82 \\
\hline Part B & 2.5 & 0.97 \\
\hline $\begin{array}{l}\text { To what extent did you find the visual effects in } \\
\text { the game psychedelic? }\end{array}$ & 3.2 & 1.23 \\
\hline $\begin{array}{l}\text { How much wilful control did you feel you had } \\
\text { over the way the visual effects changed? }\end{array}$ & 3.7 & 1.06 \\
\hline $\begin{array}{l}\text { How closely did you feel the visual effects } \\
\text { changed in relation to your brain activity? }\end{array}$ & 3.4 & 1.58 \\
\hline How immersive did you find the video game? & $\begin{array}{l}\mid \\
\text { Did wearing the EEG headset make you feel more } \\
\text { immersed in the game? }\end{array}$ & \\
\hline
\end{tabular}

\section{CONCLUSION}

Our initial system demonstrates a conceptual approach for representing ASC in video games, by automating graphical and game parameters in time. There is much scope to improve the ASC simulation by utilising newer game engines and through the design of purpose-built graphical effects and sound processes based on our ASC research. Our current work focuses on the latter, and aims to develop the audio in the game to realistically reflect auditory hallucinations. 
The pilot study conducted revealed some problems in our methodology for assessing the user experience of Quake Delirium, which would need to be addressed for future evaluations of this type of system. Firstly, it became apparent that the term 'psychedelic' as used in the questionnaire was not always fully understood by the participants. As a result, the term may have been either misunderstood, or have led the participants towards a particular evaluation or interpretation of the system. In a follow up study (publication forthcoming), we have sought to address this by exploring the repertory grid interviewing technique for analysing the experience of game audio. This technique allows participants to suggest their own terminology, thus removing the risk of leading the participant by suggesting terms such as 'psychedelic'. It is expected that using approaches such as this, we will be able to measure the extent to which simulations such as Quake Delirium are considered 'psychedelic' (or otherwise) by participants more effectively.

Along similar lines, 'immersion' is also difficult to gauge; other researchers are also currently developing new methodologies to address this [7]. It is possible that the repertory grid method may also be useful in this regard and/or other systems for recording engagement with manual user input or biosensors.

In combination with the above, the overall difficulty in measuring the effectiveness of consumer-grade EEG as a controller is that the passive nature of the experience can inherently be quite subtle. It is possible that the nature of the device as a passive or reactive controller only emerges for the user after a period of acclimatisation, once they have become familiar with the device and the way it affects the game. In a small-scale pilot study such as this, where participants had never used a device such as this before, and had a relatively short time frame in which to evaluate it, it is quite likely that these subtleties could be lost.
The problems identified mean that a substantially revised methodology will be needed for future evaluations of user experience for systems such as Quake Delirium. Nonetheless from what the pilot study seems to suggest, while the use of EEG to control psychedelic visual effects is conceptually appealing, the current system would also need to be improved to provide a more tangible connection between the headset and the ASC effects in the game. It may be possible to address this by improving the quality of the device, the methods of interpretation, or providing an improved training phase [8]. Alternatively, other types of biofeedback, affective systems [9] or sensor technology could be explored as ways to provide the passive interaction that is sought.

\section{REFERENCES}

[1] J. Weinel, "Quake Delirium: Remixing Psychedelic Video Games", Sonic Ideas/Ideas Sonicas 3(2), 2010.

[2] T.C. Demarque, E.S. Lima, "Auditory Hallucinations: Audiological Perspective for Horror Games”. SBC - Proceedings of SBGames 2013, São Paulo, Brazil

[3] A. Blake, "Top 10 Hallucinations in Video Games", Leviathyn, July $26^{\text {th }}$ 2013, available online: http://leviathyn.com/opinion/2013/07/26/top-10hallucinations-in-video-games/ [Accessed: 7th May 2014]

[4] J. Weinel, "Quake Delirium Revisited: Systems for Video Game ASC Simulations", Proceedings of the the Fifth International Conference on Internet Technologies \& Applications, Glyndŵr University, Wales, 2013

[5] T.O. Zander, C. Kothe, S. Jatzev, M. Gaertner, "Enhancing HumanComputer Interaction with input from active and passive BrainComputer Interfaces", Brain-Computer Interfaces, Spring, London, pp.181-199.

[6] J. Weinel, S. Cunningham, N. Roberts, S. Roberts, D. Griffiths, "EEG as a Controller for Psychedelic Visual Music in an Immersive Dome Environment", British Computer Society Proceedings of Electronic Visualisations and the Arts (EVA), London, 2014.

[7] H.P. Gasselseder, "Dynamic music and immersion in the actionadventure an empiral investigation", Proceedings of the $9^{\text {th }}$ Audio Mostly: A Conference on Interaction With Sound, Aalborg, Denmark, 2014.

[8] E. Miranda, S. Durrant, T. Anders, "Towards Brain-Computer Music Interfaces: Progress and Challenges", Proceedings of the International Symposium on Applied Sciences in Biomedical and Communication Technologies (ISABEL2008), Aalborg, Denmark, 2008.

[9] R. Picard, Affective Computing, MIT Press, 2000. 